'Unidad de Neurofisiología. Hospital de Carabineros de Chile. Santiago, Chile. ${ }^{2}$ Liga Chilena Contra la Epilepsia. Santiago, Chile. 3Unidad de Neurofisiología. Clínica Alemana de Santiago. Chile.

Fuente de apoyo financiero: No se recibió ninguna clase de financiamiento.

Recibido el 19 de mayo de 2016, aceptado el 12 de octubre de 2016.

Correspondencia a: Dr. Álvaro Velásquez Avenida Simón Bolivar 2200, Nunñoa, Santiago de Chile. alvarovn@gmail.com

\section{Catatonia ictal en el Servicio de Urgencia: una rara forma de presentación del estado epiléptico no convulsivo}

\author{
GERMÁN GAETE ${ }^{1,2}$, ÁLVARO VELÁSQUEZ ${ }^{1,2,3}$
}

\section{Ictal catatonia presentation as a non-convulsive status epilepticus. A case report}

The differential diagnosis of non-convulsive status epilepticus (NCSE) is often complex due to a wide clinical variability of its presentation, including psychiatric manifestations. We report a 68 years old male with a history of depression treated with venlafaxine, mirtazapine, quetiapine and risperidone, presenting in the emergency room with confusion and generalized rigidity. A brain CT scan did not show lesions. A neuroleptic syndrome was initially suspected. At the third day the obtundation worsened and an electroencephalogram (EEG) was performed, which showed epileptiform abnormalities. Treatment with valproic acid resulted in disappearance of such abnormalities. After three weeks of mechanical ventilation, the patient was extubated and remained lucid and partially orientated in time and space.

(Rev Med Chile 2017; 145: 126-130)

Key words: Catatonia; Electroencephalography; Emergency Nursing; Status Epilepticus. $\longrightarrow$ onocer el estado epiléptico no convulsivo (EENC) para el neurólogo, especialmente en el servicio de urgencia (SU), es de suma importancia. Este cuadro puede confundirse con otras patologías que presentan manifestaciones clínicas similares, con las consiguientes implicaciones terapéuticas y pronósticas. Lo diverso de estas presentaciones se refleja en una mayor dificultad en el diagnóstico diferencial, especialmente con entidades psiquiátricas, las que muchas veces son indistinguibles utilizando solo criterios clínicos.

Las actuales definiciones del EENC propuestas, primero por Meierkord y Holtkamp, y luego por Maganti et al, describen una alteración prolongada en el nivel de conciencia, asociada a actividad epileptiforme continua o descargas eléctricas prolongadas en el electroencefalograma $(\mathrm{EEG})^{1,2}$. La simultaneidad de ambos fenómenos, cambios clínicos junto con los electroencefalográficos, son la clave del diagnóstico del EENC y permiten diferenciarlo de entidades no epilépticas con manifestaciones clínicas similares.

En un estudio realizado para determinar la frecuencia en la solicitud de EEG en el SU y la Unidad de Cuidados Intensivos (UCI), la principal razón para solicitar un EEG fue el compromiso de conciencia sin causa explicable, representando el $36,3 \%{ }^{3}$. El empleo del EEG en el SU cobra importancia si consideramos que entre el $2 \%$ y el $10 \%$ de los pacientes que acuden al SU en Estados Unidos presentan algún grado de alteración en el estado mental. Si bien la mayoría de las causas son reconocibles y tratadas rápidamente, el EEG es fundamental en el diagnóstico diferencial de las crisis no epilépticas y el EENC ${ }^{4}$. El diagnóstico oportuno facilita el manejo agudo, evita procedimientos diagnósticos innecesarios, largas hospitalizaciones y reduce la morbilidad. 
En la literatura son escasos los reportes de catatonia como forma de presentación de un EENC. Las primeras descripciones corresponden a Goldensohn y Gold, luego a Thompson y Greenhouse en la década del $60^{5,6}$. Posteriormente uno de los primeros reportes de casos corresponde a Gómez et al., en 1982, quienes describen un paciente que ingresó en estupor catatónico al SU, luego de la administración de neurolépticos de alta potencia ${ }^{7}$. Lim, también en la década del 80, describe tres pacientes con catatonia ictal como manifestación de un EENC con respuesta favorable a la administración de fenitoína endovenosa ${ }^{8}$.

Presentamos un paciente con catatonia ictal en el SU, su semiología, correlato electroencefalográfico concomitante y respuesta al tratamiento con fármacos antiepilépticos.

\section{Paciente y Método}

Varón de 68 años de edad, sin antecedentes de epilepsia, en control con psiquiatra por depresión en tratamiento con venlafaxina $225 \mathrm{mg}$, mirtazapina $30 \mathrm{mg}$, quetiapina $200 \mathrm{mg}$ y risperidona 3 mg al día.

Tres días antes de su ingreso al SU tuvo control con psiquiatra, quien lo describe orientado en tiempo y espacio, autovalente y sin signos de impregnación. En dicho control redujo risperidona y mantuvo resto de terapia. Al día siguiente inició cambios conductuales, anorexia y paulatina disminución de la actividad motora, no reconoce a su familia y presenta alucinaciones visuales.
$\mathrm{Al}$ ingreso al SU se encontraba afebril y hemodinámicamente estable. Evaluado por neurólogo, se describe confuso, con rigidez generalizada. La tomografía computarizada cerebral no mostró lesiones agudas y estudios de laboratorio para causas tóxico-metabólica, incluida creatin-kinasa, fueron normales. La evaluación psiquiátrica consignó mutismo y rigidez cérea planteando síndrome catatónico en paciente con depresión mayor y como diagnóstico diferencial de síndrome neuroléptico. Se indicó traslado a clínica psiquiátrica donde no fue recibido.

Al tercer día en SU, empeora, describiéndose sopor profundo. Se realizó EEG (Figura 1) que mostró actividad de fondo lenta theta, polimorfa, generalizada no reactiva, sobre la que se observan descargas generalizadas de puntas y punta onda lenta de máximo bifrontal, con patrón pseudoperiódico a $1 \mathrm{~Hz}$, presentándose en salvas de hasta 8 segundos de duración, seguidas de periodos de depresión de voltaje (GPEDs). Se intentó realizar resonancia magnética nuclear de cerebro, no pudiendo concretarse por emesis y broncoaspiración durante examen, requiriendo sedación con midazolam, intubación y apoyo ventilatorio (VM) en UCI, recibiendo tratamiento antibiótico por neumonía aspirativa.

Dado los hallazgos de EEG se inició terapia empírica con aciclovir en espera de resultados de punción lumbar que reveló líquido cefalorraquídeo claro, látex para meningitis, PCR para herpes y enterovirus negativos. Simultáneamente inició terapia antiepiléptica, dado la necesidad del uso

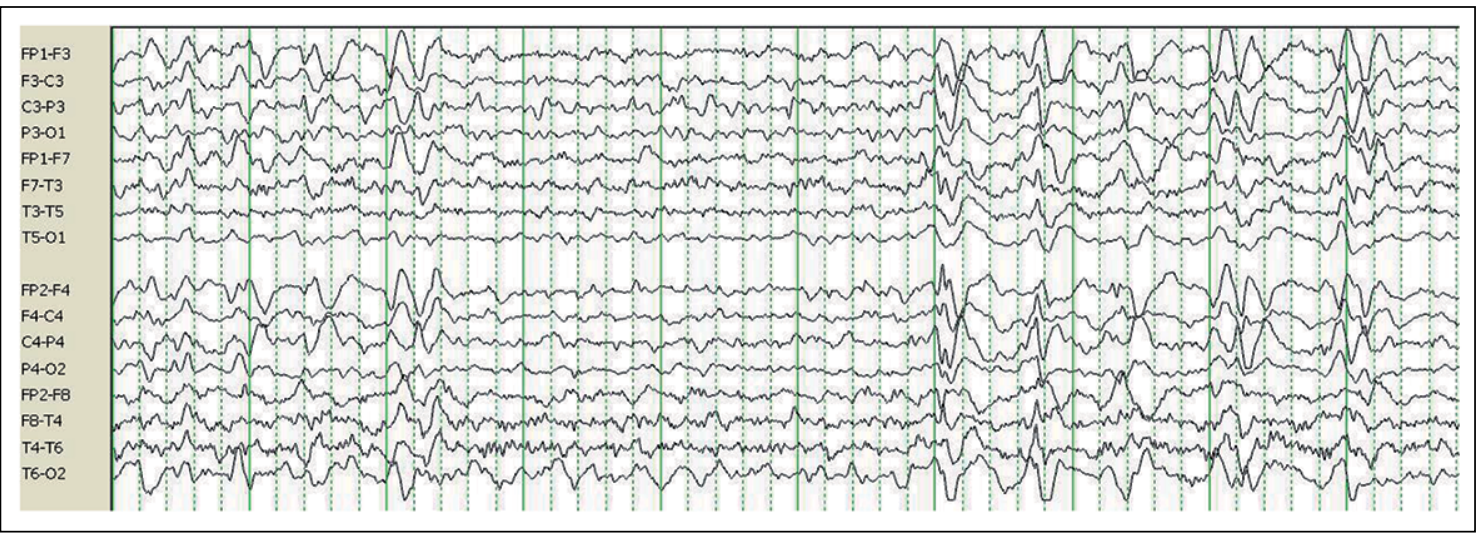

Figura 1. EEG que evidencia actividad de fondo lenta theta polimorfa generalizada no reactiva, se agregan frecuentes descargas generalizadas de puntas y punta onda lenta de máximo bifrontal que adopta un patrón pseudoperiódico a 1 Hz y se presentan en salvas de hasta 8 segundos de duración seguidas de breves periodos de depresión de voltaje (GPEDs). 


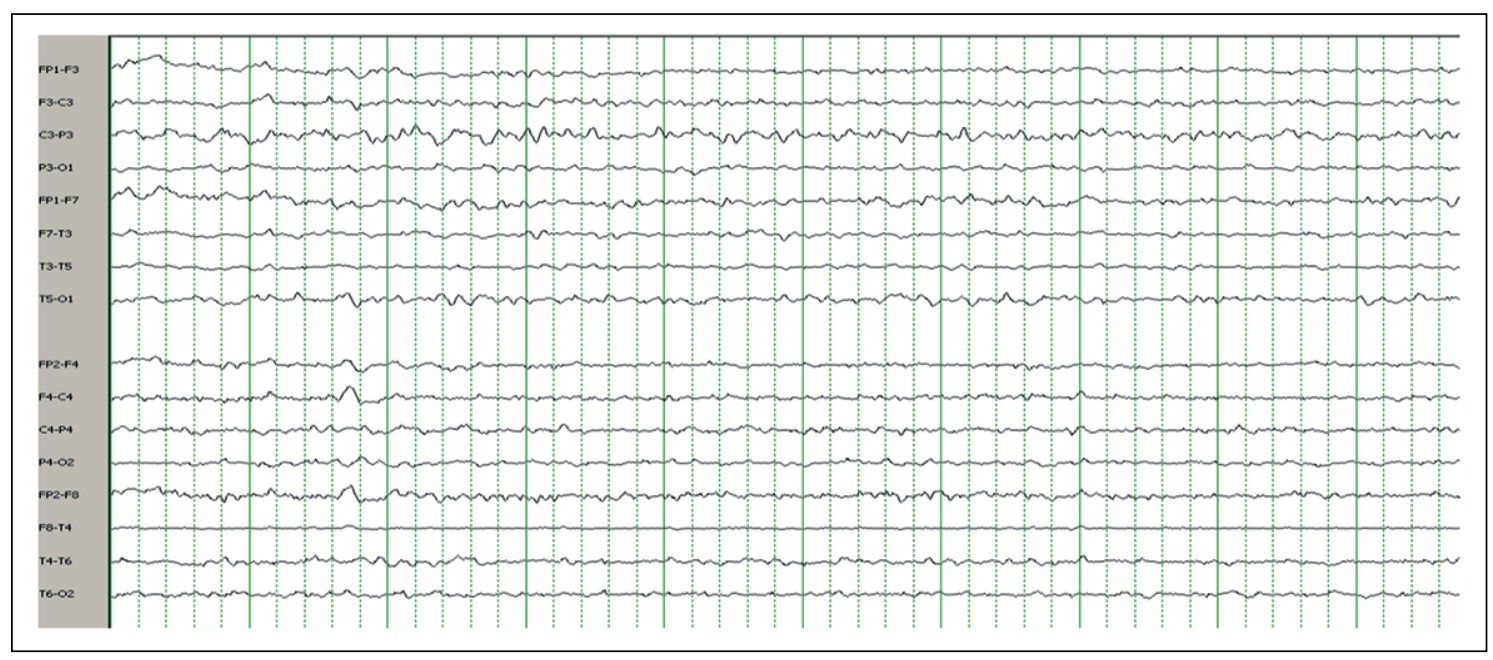

Figura 2. EEG post carga de AV. Actividad de base theta con supresión de la actividad epileptiforme previamente visualizada.

de midazolam para la conexión a ventilación mecánica se decide iniciar acido valproico (AV). Al cuarto día de tratamiento con AV, el EEG de control (Figura 2) mostró actividad de base lenta en rango theta generalizada y ausencia de actividad epileptiforme. Clínicamente, permaneció en $\mathrm{VM}$, adquirió vigilia al estímulo, siguiendo con la mirada y obedeciendo órdenes simples. Por insuficiencia respiratoria estuvo 3 semanas en VM. Tras la extubación, se realizó control de EEG que fue normal. Clínicamente, parcialmente orientado en tiempo y espacio, sin otros déficits.

\section{Discusión}

El EENC es una entidad subdiagnosticada en el SU, tanto porque no se reconoce el cuadro clínico como por la dificultad para realizar un EEG que lo confirme. La anamnesis y el examen físico pueden ser indistinguibles de otras entidades e incluso desorientar al médico de urgencia, como lo ocurrido con nuestro paciente donde la historia de depresión previa y los hallazgos de "rigidez cérea" y "mutismo" en el examen físico generaron que se solicitará evaluación psiquiátrica demorando el diagnóstico definitivo.

Se han descrito criterios clínicos con el fin de aumentar la sensibilidad diagnóstica tales como la pesquisa de factores de riesgo para desarrollar un foco epileptógeno (accidente cerebrovascular, neurocirugía, tumor, meningoencefalitis) junto con algunas alteraciones en el examen físico, principalmente oculomotoras (nistagmos, parpadeo rítmico, desviación ocular mantenida) y neurovegetativas como la sudoración profusa ${ }^{10}$, sin embargo, la certeza diagnóstica requiere del EEG, que en nuestro caso tardó tres días en ser solicitado, período en que el paciente presentó neumonía por aspiración, complicación ampliamente descrita asociada al compromiso de conciencia ${ }^{11}$ que podría haberse evitado disminuyendo el tiempo de hospitalización.

Una de las principales causas del EENC es la suspensión de la terapia antiepiléptica sin embargo, el 70\% de los mayores de 60 años que presentan un EENC no tiene una epilepsia conocida, proporción que se invierte a edades más tempranas ${ }^{12}$. En esta población las causas son otras y una vez descartado el daño encefálico o metabólico siempre debe sospecharse acción de fármacos, tal como el caso del EENC por retiro brusco de benzodiacepinas ${ }^{13}$. Se describen también en relación al tratamiento con antidepresivos ${ }^{14,15}$ $y$ neurolépticos ${ }^{16}$. Nuestro paciente no presentaba historia de epilepsia y tanto los estudios de imágenes como de laboratorio no revelaron alteraciones significativas, aunque se encontraba en tratamiento con altas dosis de antidepresivos y neurolépticos por un cuadro depresivo mayor. Asumimos que la combinación de estos fármacos generó el EENC en nuestro paciente. 
Variados fueron los intentos por definir las características electroencefalográficas del EENC. Sin una definición universalmente aceptada, existe actualmente cierto grado de consenso sobre algunos patrones "característicos". Especialmente importantes son los patrones de EEG periódicos, si bien siguen siendo motivo de debate, muchos autores sugieren que son inequívocamente epilépticos en algunos casos. Estos patrones incluyen la actividad delta rítmica, ondas trifásicas generalizadas, las descargas epileptiformes periódicas lateralizadas, descargas epileptiformes periódicas generalizadas (GPEDs), las descargas epileptiformes periódicas con lateralización bilateral independientes (BIPLEDs), y el estímulo inducido por descargas rítmicas, periódica o ictal (SIRPIDs). Los patrones periódicos se ven en una amplia variedad de etiologías, por lo tanto, los pacientes deberán ser estudiados cuidadosamente para enfermedades infecciosas, tóxico-metabólicas y/o lesiones intracraneales considerando siempre el EENC en el diagnóstico diferencial ${ }^{1,2,17-19}$. El EEG en nuestro paciente coincide con lo descrito en la literatura e igual que los escasos reportes de casos de catatonia ictal donde se documentó adecuadamente la evolución "electroclínica", nuestro paciente presentó una mejoría tanto clínica como en el EEG posterior al inicio del tratamiento antiepiléptico.

El tratamiento del EENC sigue siendo controversial. No hay actualmente evidencia científica de ensayos clínicos randomizados sobre un tratamiento establecido para el EENC por lo que las recomendaciones están basadas en la experiencia clínica y estudios de casos. Nosotros decidimos el empleo de AV basados en la experiencia del uso de este fármaco en nuestro centro y estudios que postulan una mayor eficacia y seguridad con $\mathrm{AV}$ en este tipo de pacientes ${ }^{20}$. Al asumir un mecanismo de acción GABAérgico, el AV sería de primera elección en el manejo del EENC porque abarcaría todos los subtipos clínicos de este, sin el riesgo de empeorar algunos, como se describe para los bloqueadores de canales de sodio. Además, es mejor tolerado, tiene menos efectos adversos y el manejo sería más sencillo.

\section{Conclusión}

Esperamos que nuestra experiencia aporte en el reconocimiento de manifestaciones clínicas inusuales del EENC y recomendamos realizar un EEG frente a cualquier alteración en el estado mental sin causa explicable.

Remarcamos la importancia del estudio y reconocimiento de ciertos patrones en el EEG sugerentes de EENC, con el fin de unificar criterios en su interpretación, aunque sabemos, muchas veces esto no es posible y lo que para un intérprete pareciera un patrón de EEG de EECN, no lo es para otro, lo cual refleja el "arte" de la interpretación del EEG ${ }^{17}$.

Finalmente, frente a la sospecha clínica de EENC y en presencia de un electroencefalograma compatible, entendiendo que los patrones eléctricos aún son motivo de controversia, recomendamos prueba clínica con antiepilépticos.

\section{Referencias}

1. Meierkord H, Holtkamp M. Non-convulsive status epilepticus in adults: clinical forms and treatment. Lancet Neurol 2007; 6 (4): 329-39.

2. Maganti R, Gerber P, Drees C, Chung S. Nonconvulsive status epilepticus. Epilepsy Behav 2008; 12 (4): 572-86.

3. Ricardo JA, França MC Jr, Lima FO, Yassuda CL, Cendes F. The impact of EEG in the diagnosis and management of patients with acute impairment of consciousness. Arq Neuropsiquiatr 2012 Jan; 70 (1): 34-9.

4. Zehtabchi S, Abdel Baki SG, Malhotra S, Grant AC Nonconvulsive seizures in patients presenting with altered mental status: an evidence-based review. Epilepsy Behav 2011 Oct; 22 (2): 139-43.

5. Goldensohn ES, Gold AP. Prolonged behavioral disturbances as ictal phenomena. Neurology 1960; 10: 1-9.

6. Thompson SW, Greenhouse AH. Petit mal status in adults. Ann Intern Med 1968; 68 (6): 1271-9.

7. Gómez EA, Comstock BS, Rosario A. Organic versus etiology in catatonia: case report. J Clin Psychiatry 1982 May; 43 (5): 200-1.

8. Lim J, Yagnik P, Schraeder P, Wheeler S. Ictal catatonia as a manifestation of nonconvulsive status epilepticus. J Neurol Neurosurg Psychiatry 1986; 49 (7): 833-6.

9. Kanemoto K, Miyamoto T, Abe R. Ictal catatonia as a manifestation of de novo absence status epilepticus following benzodiazepine withdrawal. Seizure 1999 Sep; 8 (6): 364-6.

10. Husain AM, Horn GJ, Jacobson MP. Non-convulsive status epilepticus: usefulness of clinical features in selecting patients for urgent EEG. J Neurol Neurosurg Psychiatry 2003; 74 (2): 189-91.

11. Petroianni A, Ceccarelli D, Conti V, Terzano C. Aspira- 
tion pneumonia. Pathophysiological aspects, prevention and management. A review. Panminerva Med 2006 Dec; 48 (4): 231-9.

12. De Lorenzo RJ, Hauser WA, Towne AR, Boggs JG, Pellock JM, Penberthy L, et al. A prospective, population-based epidemiologic study of status epilepticus in Richmond, Virginia. Neurology 1996; 46 (4): 1029-35.

13. Thomas P, Beaumanoir A, Genton P, Dolisi C, Chatel M. "De novo" absence status of late onset: report of 11 cases. Neurology 1992; 42 (1): 104-10.

14. Yoshino A, Watanabe M, Shimizu K, Goto T, Ichinowatari N, Yoshimasu H, et al. Nonconvulsive status epilepticus during antidepressant treatment. Neuropsychobiology 1997; 35 (2): 91-4.

15. Miyata H, Kubota F, Shibata N, Kifune A. Non-convulsive status epilepticus induced by antidepressants. Seizure 1997; 6 (5): 405-7.

16. Yoshino A, Yoshimasu H, Tatsuzawa Y, Asakura T,
Hara T. J Clin Psychopharmacol. Non convulsive status epilepticus in two patients with neuroleptic malignant syndrome. 1998 Aug; 18 (4): 347-9.

17. Sutter R, Kaplan PW. Electroencephalographic criteria for nonconvulsive status epilepticus: synopsis and comprehensive survey. Epilepsia 2012 Aug; 53 Suppl 3: $1-51$.

18. Liberalesso PB, Garzon E, Yacubian EM, Sakamoto AC. Refractory non convulsive status epilepticus in coma: analysis of the evolution of ictal patterns. Arq Neuropsiquiatr 2012 Jul; 70 (7): 501-5.

19. Andraus ME, Andraus CF, Alves-Leon SV. Periodic EEG patterns: importance of their recognition and clinical significance. Arq Neuropsiquiatr 2012 Feb; 70 (2): 14551.

20. Sinha S, Naritoku DK. Intravenous valproate is well tolerated in unstable patients with status epilepticus. Neurology 2000; 55: 722-4. 